\title{
Effect of an error in the forward difference based tabular values of the rate of enzymatic reaction based mathematical functions
}

\author{
Nizam Uddin $^{1 *}$, Saad Mohammed ${ }^{2}$ \\ ${ }^{I}$ M. B. Khalsa College, Indore-452002, Madhya Pradesh, India \\ ${ }^{2}$ Mahakal Institute of Pharmaceutical Studies, Behind Air Strip, Ujjain-456664, Madhya Pradesh, India. \\ *Corresponding author E-mail: nizamuddin4research@gmail.com
}

\begin{abstract}
In this research paper, we explained the effect of an error in the tabular values of substrate concentration, enzyme concentration and temperature with the rate of enzymatic reaction. All values are in interval. These values are obtained by mathematical functions of the rate of enzymatic reaction. Mathematical functions show that the concentration of substrate is the limiting factor, as the substrate concentration increases, the Enzyme reaction rate increases. Assuming a sufficient concentration of substrate is available, increasing Enzyme concentration will increase the rate of enzymatic reaction. Temperature, concentration of substrate and concentration of enzyme are increased the rate of enzymatic reaction at a limit which is called optimum limit. We take these values in tabular form with forward difference and added an error in the tabular values of substrate concentration, enzyme concentration and temperature with the rate of enzymatic reaction. Tabular values show the erroneous values. There are changes in tabular values. The effect of an error goes increasing with the succession. The coefficients of " $\varepsilon$ " are the binomial coefficients with alternating signs. In various difference columns of the difference table, the algebraic sum of the error is zero. The maximum error occurs in the same line as the erroneous tabular value. This is show the effect of tabular values of substrate concentration, enzyme concentration and temperature with the rate of enzymatic reaction.
\end{abstract}

Keywords: Effect of an error, Forward difference, Mathematical functions, Rate of Enzymatic Reaction, Tabular values.

\section{Introduction}

The forward difference is a finite difference which is the measure of the difference between the current term and the next [1]-[3]. Mathematical functions are defined the rate of enzymatic reaction [1]. $V=f(S)$ is defined the rate of rate of enzymatic reaction with concentration of substrate, $V=f(E)$ is defined the rate of rate of enzymatic reaction with concentration of enzyme and $V=f(T)$ is defined the rate of rate of enzymatic reaction with temperature[4-6]. These mathematical functions are show that the concentration of substrate is the limiting factor, as the substrate concentration increases, the Enzyme reaction rate increases. Assuming a sufficient concentration of substrate is available, increasing Enzyme concentration will increase the rate of enzymatic reaction. Temperature, concentration of substrate and concentration of enzyme are increased the rate of enzymatic reaction at a limit which is called optimum limit [6]-[10]

\section{Effect of an error in the tabular value of $V=f(S)$}

Suppose $f\left(S_{0}\right), f\left(S_{1}\right), f\left(S_{2}\right), f\left(S_{3}\right), \ldots, f\left(S_{n}\right)$ are the true value of the function $f(S)$ and suppose that the value $f\left(S_{4}\right)$ is in error by quantity $\varepsilon$ so that erroneous value of $f\left(S_{4}\right)$ is $f\left(S_{4}\right)+\varepsilon$. Then the table will be as follow: 


\begin{tabular}{|l|l|l|l|}
\hline$f\left(S_{0}\right)$ & & & \\
$f\left(S_{1}\right)$ & $\Delta f\left(S_{0}\right)$ & & \\
$f\left(S_{2}\right)$ & $\Delta f\left(S_{1}\right)$ & $\Delta^{2} f\left(S_{0}\right)$ & \\
$f\left(S_{3}\right)$ & $\Delta f\left(S_{2}\right)$ & $\Delta^{2} f\left(S_{1}\right)$ & $\Delta^{3} f\left(S_{0}\right)$ \\
$f\left(S_{4}\right)+\varepsilon$ & $\Delta f\left(S_{3}\right)+\varepsilon$ & $\Delta^{2} f\left(S_{2}\right)+\varepsilon$ & $\Delta^{3} f\left(S_{1}\right)+\varepsilon$ \\
$f\left(S_{5}\right)$ & $\Delta f\left(S_{4}\right)-\varepsilon$ & $\Delta^{2} f\left(S_{3}\right)-2 \varepsilon$ & $\Delta^{3} f\left(S_{2}\right)-3 \varepsilon$ \\
$f\left(S_{6}\right)$ & $\Delta f\left(S_{5}\right)$ & $\Delta^{2} f\left(S_{4}\right)+\varepsilon$ & $\Delta^{3} f\left(S_{3}\right)+3 \varepsilon$ \\
$f\left(S_{7}\right)$ & $\Delta f\left(S_{6}\right)$ & $\Delta^{2} f\left(S_{5}\right)$ & $\Delta^{3} f\left(S_{4}\right)-\varepsilon$ \\
\hline
\end{tabular}

\section{Effect of an error in the tabular value of $V=f(E)$}

Suppose $f\left(E_{0}\right), f\left(E_{1}\right), f\left(E_{2}\right), f\left(E_{3}\right), \ldots, f\left(E_{n}\right)$ are the true value of the function $f(E)$ and suppose that the value $f\left(E_{4}\right)$ is in error by quantity $\varepsilon$ so that erroneous value of $f\left(E_{4}\right)$ is $f\left(E_{4}\right)+\varepsilon$. Then the table will be as follows:

\begin{tabular}{|l|l|l|l|}
\hline$f\left(E_{0}\right)$ & & & \\
$f\left(E_{1}\right)$ & $\Delta f\left(E_{0}\right)$ & & \\
$f\left(E_{2}\right)$ & $\Delta f\left(E_{1}\right)$ & $\Delta^{2} f\left(E_{0}\right)$ & \\
$f\left(E_{3}\right)$ & $\Delta f\left(E_{2}\right)$ & $\Delta^{2} f\left(E_{1}\right)$ & $\Delta^{3} f\left(E_{0}\right)$ \\
$f\left(E_{4}\right)+\varepsilon$ & $\Delta f\left(E_{3}\right)+\varepsilon$ & $\Delta^{2} f\left(E_{2}\right)+\varepsilon$ & $\Delta^{3} f\left(E_{1}\right)+\varepsilon$ \\
$f\left(E_{5}\right)$ & $\Delta f\left(E_{4}\right)-\varepsilon$ & $\Delta^{2} f\left(E_{3}\right)-2 \varepsilon$ & $\Delta^{3} f\left(E_{2}\right)-3 \varepsilon$ \\
$f\left(E_{6}\right)$ & $\Delta f\left(E_{5}\right)$ & $\Delta^{2} f\left(E_{4}\right)+\varepsilon$ & $\Delta^{3} f\left(E_{3}\right)+3 \varepsilon$ \\
$f\left(E_{7}\right)$ & $\Delta f\left(E_{6}\right)$ & $\Delta^{2} f\left(E_{5}\right)$ & $\Delta^{3} f\left(E_{4}\right)-\varepsilon$ \\
\hline
\end{tabular}

\section{Effect of an error in the tabular value of $V=f(T)$}

Suppose $f\left(T_{0}\right), f\left(T_{1}\right), f\left(T_{2}\right), f\left(T_{3}\right), \ldots, f\left(T_{n}\right)$ are the true value of the function $f(T)$ and suppose that the value $f\left(T_{4}\right)$ is in error by quantity $\varepsilon$ so that erroneous value of $f\left(T_{4}\right)$ is $f\left(T_{4}\right)+\varepsilon$. Then the table will be as follows:

\begin{tabular}{|l|l|l|l|}
\hline$f\left(T_{0}\right)$ & & & \\
$f\left(T_{1}\right)$ & $\Delta f\left(T_{0}\right)$ & & \\
$f\left(T_{2}\right)$ & $\Delta f\left(T_{1}\right)$ & $\Delta^{2} f\left(T_{0}\right)$ & \\
$f\left(T_{3}\right)$ & $\Delta f\left(T_{2}\right)$ & $\Delta^{2} f\left(T_{1}\right)$ & $\Delta^{3} f\left(T_{0}\right)$ \\
$f\left(T_{4}\right)+\varepsilon$ & $\Delta f\left(T_{3}\right)+\varepsilon$ & $\Delta^{2} f\left(T_{2}\right)+\varepsilon$ & $\Delta^{3} f\left(T_{1}\right)+\varepsilon$ \\
$f\left(T_{5}\right)$ & $\Delta f\left(T_{4}\right)-\varepsilon$ & $\Delta^{2} f\left(T_{3}\right)-2 \varepsilon$ & $\Delta^{3} f\left(T_{2}\right)-3 \varepsilon$ \\
$f\left(T_{6}\right)$ & $\Delta f\left(T_{5}\right)$ & $\Delta^{2} f\left(T_{4}\right)+\varepsilon$ & $\Delta^{3} f\left(T_{3}\right)+3 \varepsilon$ \\
$f\left(T_{7}\right)$ & $\Delta f\left(T_{6}\right)$ & $\Delta^{2} f\left(T_{5}\right)$ & $\Delta^{3} f\left(T_{4}\right)-\varepsilon$ \\
\hline
\end{tabular}




\section{Conclusion}

The effect of an error goes on increasing with the succession in the tabulated values of the rate of enzymatic reaction. The coefficients of $\varepsilon$ are the binomial coefficients with alternating signs. In various difference columns of the difference table, the algebraic sum of the error is zero. The maximum error occurs in the same line as the erroneous tabular value. With these points in mind we will try to locate the error. In case there are two or more errors, the successive differences will be irregular and it will not be possible to locate the errors in the tabular values of the mathematical functions. These errors in tabulated values of the mathematical functions with temperature, concentration of substrate and concentration of enzyme are affected the forward difference of the rate of enzymatic reaction.

\section{References}

[1] Abramowitz, M. and Stegun, I. A. (Eds.). Handbook of Mathematical Functions with Formulas, Graphs, and Mathematical Tables, 9th printing. New York: Dover, p. 877, 1972.

[2] Sloane, N. J. A. and Plouffe, S. The Encyclopedia of Integer Sequences. San Diego, CA: Academic Press, p. $10,1995$.

[3] Zdeněk SMÉKAL, Difference Equations with Forward and Backward Differences and Their Usage in Digital Signal Processor Algorithms. RADIOENGINEERING, 15( 2),45-52, 2006

[4] Nizam Uddin, Enzyme Concentration, Substrate Concentration and Temperature based Formulas for obtaining intermediate values of the rate of enzymatic reaction using Lagrangian polynomial. International Journal of Basic And Applied Sciences, 1(3), 299-302, 2012.

[5] Nizam Uddin, "Inverse Interpolation: The Rate of Enzymatic Reaction based Finite differences, Formulas for obtaining intermediate values of Temperature, Substrate Concentration, Enzyme Concentration and their Estimation of Errors," International Journal of Innovation and Applied Studies, vol. 3, no. 1, pp. 160-204, May 2013.

[6] Nizam Uddin, Mathematical functions of the rate of enzymatic reaction with Temperature, concentration of substrate and concentration of enzyme are proved that the divide differences are symmetrical in all their arguments. International Journal Of Advanced Chemistry, 1(1), 1-4, 2013.

[7] Nizam Uddin, "Estimation of Errors: Mathematical Expressions of Temperature, Substrate Concentration and Enzyme Concentration based Formulas for obtaining intermediate values of the Rate of Enzymatic Reaction," International Journal of Innovation and Applied Studies, vol. 2, no. 2, pp. 153-172, Feb. 2013.

[8] Nizam Uddin, "Interpolate the Rate of Enzymatic Reaction: Temperature, Substrate Concentration and Enzyme Concentration based Formulas using Newton's Method" , "International Journal of Research in Biochemistry and Biophysics", 2( 2), 5-9, 2012.

[9] Nizam Uddin, "Mathematical Expressions for Estimation of Errors in the Formulas which are used to obtaining intermediate values of Biological Activity in QSAR,” International Journal of Innovation and Applied Studies, vol. 2, no. 3, pp. 272-279, March 2013.

[10] Nizam Uddin, "Formulas for Obtaining Intermediate Values of Biological Activity in QSAR using Lagrangian polynomial and Newton's method", "Science Insights: An International Journal”, 2(4), 21-23, 2012. 